\title{
Efficacy and Tolerability of Vortioxetine in Patients with Major Depressive Disorder: A Clinical Perspective from 33 Indian Cases
}

\author{
Mansi Vora
}

\begin{abstract}
Aim: Limited data are available concerning the tolerability and efficacy of vortioxetine in Indian depressive patients. This retrospective prescription review analysis provides clinical experience of vortioxetine in patients with major depressive disorder (MDD) and high ratings of anxiety (anxious depression).

Materials and methods: This prescription data review was carried out at Westcoast Institute of Neuroscience, India. Data were extracted from prescription of all the patients suffering from depression and other psychiatric disorders who were treated with vortioxetine (Brintellix) from February 1, 2019, to May 31, 2020.

Results:Thirty-three prescription records were reviewed during the study. Vortioxetine improved depressive and psychological symptoms with minimum adverse effects. Vortioxetine showed good tolerability in majority of patients who were still receiving vortioxetine monotherapy by the end of the therapy. Mental Status Examination (MSE) revealed that the patients treated with vortioxetine demonstrated better orientation, reactiveness, and attentiveness.

Conclusion: This retrospective prescription review analysis demonstrated the effectiveness, and tolerability of vortioxetine in Indian MDD patients with depressive and anxiety symptoms.

Keywords: Antidepressant, Cognitive functions, Major depressive disorder, Prescription review, Selective serotonin reuptake inhibitor, Vortioxetine.

Indian Journal of Private Psychiatry (2020): 10.5005/jp-journals-10067-0061
\end{abstract}

\section{INTRODUCTION}

Major depressive disorder (MDD) is a chronic depressive condition characterized by high rates of functional impairment, psychiatric comorbidity, and significant social distress. The overall prevalence of MDD in India is 5.25\%, highest in the $40-59$ age groups (OR: 1.93), females (OR: 1.23) and urban cities with $>1$ million population (OR: 3.06). Apart from a negative influence on the patients' quality of life, ${ }^{2}$ MDD can substantially increase the socioeconomic burden of any region ${ }^{3,4}$ due to productivity losses in the workplaces derived by depressed individuals of working age. ${ }^{5-7}$ A previous study thus found that approximately half of the indirect costs of managing depression (constituting approximately $80 \%$ of total costs) were associated with the loss of productivity and unemployment. ${ }^{8}$

Identifying medications with efficacy across patient with MDD continues to be of clinical interest, and several studies have evaluated the effects of patient demographics, MDD history, and symptom severity on treatment outcomes. Several characteristics of a depressive patient such as older age, male gender, severity of the depression at the baseline, personal/family history of depressive episodes, and longer duration of depression can affect the efficacy of an antidepressant. ${ }^{9}$ It is estimated that during a 1-year period, only $6 \%$ of patients with MDD will achieve remission with treatment. ${ }^{10}$ Family life and social functioning along with workplace productivity are adversely impacted by the low rate of remission. ${ }^{11}$

Vortioxetine, a selective serotonin reuptake inhibitor (SSRI), produces a pleiotropic effect on level of neurotransmitters such as acetylcholine, histamine, dopamine, norepinephrine, and serotonin. ${ }^{12}$ Several meta-analyses support the efficacy and tolerability of vortioxetine at reducing severity of MDD. ${ }^{13-15} \operatorname{In} 2018$,
Westcoast Institute of Neuroscience, Mumbai, Maharashtra, India

Corresponding Author: Mansi Vora, Westcoast Institute of Neuroscience, Mumbai, Maharashtra, India, Phone: +91 9930092762 , e-mail: drmansisvora@gmail.com

How to cite this article: Vora M. Efficacy and Tolerability of Vortioxetine in Patients with Major Depressive Disorder: A Clinical Perspective from 33 Indian Cases. Ind J Priv Psychiatry 2020;14(2):57-61.

Source of support: Nil

Conflict of interest: None

United States Food and Drug Administration (USFDA) allowed the manufacturers to mention the pro-cognitive efficacy of vortioxetine on its product insert.

Although several previous studies have reported the positive efficacy of vortioxetine for treating patients with depression, limited data are available concerning the efficacy and safety of vortioxetine in Indian depressive patients. The purpose of this retrospective prescription review analysis is to assess the efficacy, and tolerability of vortioxetine in patients with MDD and high ratings of anxiety (anxious depression). We hope that this review will provide clinicians additional information to help guide them toward optimal treatment modalities for MDD maintenance therapy.

\section{Materials and Method}

This is a retrospective data review which was carried out at Westcoast Institute of Neuroscience, India. Data were extracted from the prescription of all the patients with depression and other 
psychiatric disorders who were treated with vortioxetine (Brintellix) from February 1, 2019, to May 31, 2020. Being a retrospective prescription review and deidentified data analysis, institutional ethics committee approval was not needed.

\section{Data Collection}

The prescriptions were reviewed for patient's demographics; primary diagnosis; psychological symptoms; dosing; and combination pattern of vortioxetine, adverse drug reaction, and Mental Status Examination (MSE) components. Apart from these, the Montgomery-Åsberg Depression Rating Scale (MADRS) and Hamilton Depression Rating Scale (HDRS) were used to evaluate the effect of vortioxetine on depressive symptoms, and anxiety severity. The Massachusetts General Hospital-Cognitive and Physical Functioning Questionnaire (MGH-CPFQ), a self-rated measure of cognitive and physical functioning, was administered to assess the severity of depression with convulsion. The Body Dysmorphic Disorder-Yale-Brown Obsessive-Compulsive Scale (BDD-YBOCS), an observer-rated scale, was used to assess the severity of body dysmorphic disorder.

\section{Statistical Analysis}

Parametric variables were expressed as mean \pm standard deviation (SD) or range; nonparametric variables were expressed as median, minimum, and maximum. Categorical variables were reported in percentages.

\section{Results}

\section{Demographic Data}

Thirty-three prescription records were reviewed during the study. There were a greater proportion of female patients $(60.6 \%)$. Majority of the patients belonged to the age group of 30 to 49 years (42.4\%) followed by 15 to 29 years (30.3\%) and 50 to 69 years (27.3\%). The baseline MADRS and HDRS total scores were approximately 27 and 22, respectively. Table 1 displays the baseline clinical and demographic characteristics of patients in detail.

\section{Vortioxetine Dosage and Duration of Administration}

$57.6 \%$ and $42.4 \%$ patients were started with a dose of $5 \mathrm{mg}$ and $10 \mathrm{mg}$ of vortioxetine, respectively. After a mean (SD) treatment duration of 173 (83.1) days, 45.5\%,33.3\%, and $21.2 \%$ of patients were receiving a dose of 5,10 , and $20 \mathrm{mg}$, respectively. With respect to the dual or triple combination group, the most prescribed antidepressant with vortioxetine was amitriptyline and escitalopram. At the start of the therapy, $42.4 \%$ of patients were receiving vortioxetine in combination with another antidepressant. However, by the end of the therapy, more than $72 \%$ of patients were receiving vortioxetine monotherapy, while the combination therapy was provided to only $27 \%$ of patients (Fig. 1).

The changes of MADRS, HDR-S, MGH-CPFQ, and BDD-YBOCS total scores from baseline to the endpoint were $-20.25,-18,-14$, and -23 , respectively (Fig. 2). Each assessment score was decreased by $77.6 \%, 81.8 \%, 56.3 \%$, and $69.7 \%$, respectively, from baseline to the endpoint.

\section{Psychological Symptoms}

An improvement in depressive symptoms such a low mood, sleep disturbance, dizziness, lack of interest, loss of appetite, lassitude, rumination, volition, biofunctional disturbance, sharpness,

\begin{tabular}{lc}
\multicolumn{2}{l}{ Table 1: Summary of baseline characteristics of the subjects $(n=33)$} \\
\hline Parameters & Data \\
\hline Age (year); mean \pm SD & $40.85 \pm 14.47$ \\
Age group; $n$ (\%) & \\
15-29 & $10(30.3)$ \\
$30-49$ & $14(42.4)$ \\
$50-69$ & $9(27.3)$ \\
Sex, female; $n$ (\%) & $20(60.6 \%)$ \\
Education; $n$ (\%) & \\
$\quad$ Middle school & $12(36.4 \%)$ \\
ZHigh school & $21(63.6 \%)$ \\
Living status, married; $n$ (\%) & $16(48.5 \%)$ \\
Primary diagnosis; $n$ (\%) & \\
Anxiety & $4(12.1)$ \\
Major depressive disorder (MDD) & $21(63.6)$ \\
Mixed anxiety depression & $4(12.1)$ \\
Body dysmorphic disorder & $1(3.0)$ \\
Depression with convulsion & $3(9.1)$ \\
Psychometric assessment; mean \pm SD & \\
MADRS & $27 \pm 3$ \\
HDRS & $22 \pm 3$ \\
MGH-CPFQ & $26 \pm 3$ \\
BDD-YBOCS & 33 \\
\hline
\end{tabular}

MADRS: Montgomery-Åsberg Depression Rating Scale; HDRS: Hamilton Depression Rating Scale; MGH-CPFQ: Massachusetts General Hospital-Cognitive and Physical Functioning Questionnaire; BDD-YBOCS: Body Dysmorphic Disorder-Yale-Brown Obsessive-Compulsive Scale

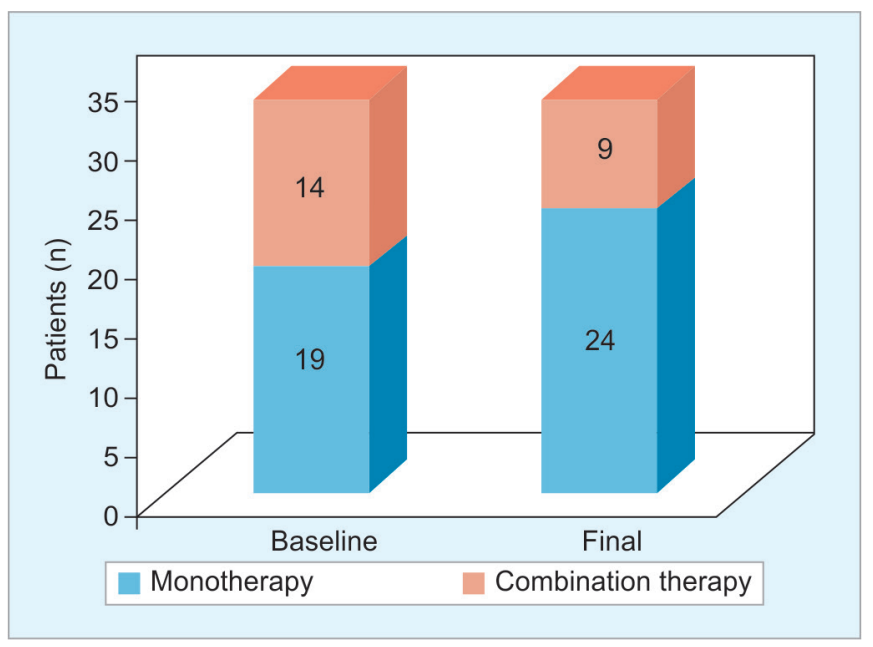

Fig. 1: Changes in therapeutic regimen of vortioxetine from baseline to the end of the therapy

cognition, and interpersonal disabilities was observed from baseline to the end of the treatment (Table 2).

\section{Adverse Effects}

Constipation (21.2\%) was the most commonly observed adverse effect of vortioxetine therapy followed by gastritis (12.1\%), pruritus $(9.1 \%)$, erectile dysfunction (6.1\%), itching (3\%), and hair fall (3\%) (Fig. 3).

\section{Mental Status Examination (MSE)}

The MSE of patients treated with vortioxetine is presented in Table 3. Nonreactiveness in $48.5 \%$ and disorientation in $33.33 \%$ 


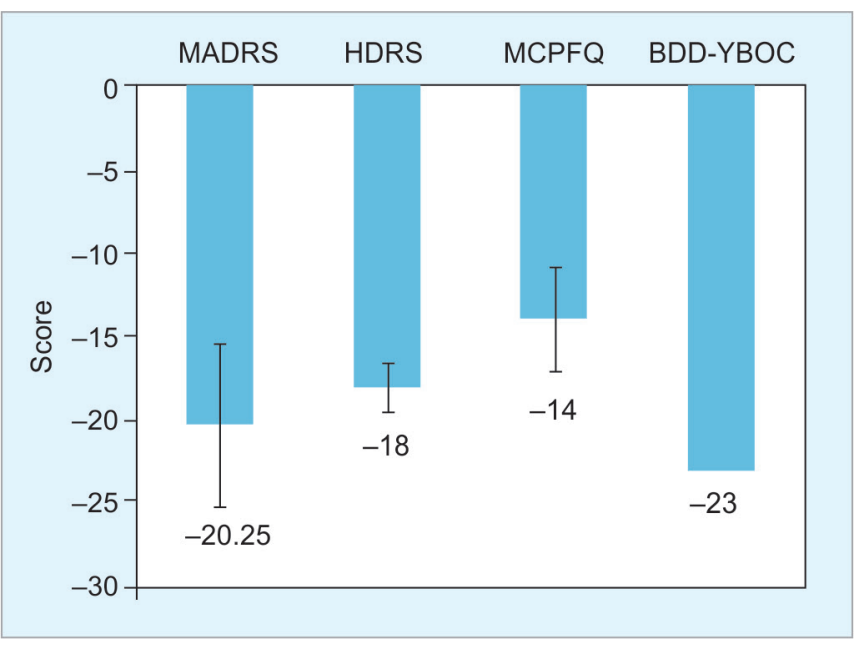

Fig. 2: Changes of outcome measures from baseline in the study. Data represent the mean \pm SD from baseline at each psychometric scale

Table 2: Psychological symptoms

\begin{tabular}{llcl}
\hline Si. No. & Parameters & $\begin{array}{l}\text { Baseline } \\
\text { symptoms } \\
n(\%)\end{array}$ & $\begin{array}{l}\text { Final } \\
\text { symptoms } \\
n(\%)\end{array}$ \\
\hline 1 & Low mood present & $27(81.82)$ & 0 \\
2 & Dizziness present & $4(12.12)$ & 0 \\
3 & Lack of interest present & $17(51.51)$ & 0 \\
4 & Loss of appetite & $8(24.24)$ & $1(3.03)$ \\
5 & Lassitude present & $14(42.42)$ & $2(6.06)$ \\
6 & Rumination present & $10(30.3)$ & $2(6.06)$ \\
7 & Volition absent & $19(57.57)$ & $3(9.09)$ \\
8 & Biofunctional disturbance & $15(45.45)$ & $3(9.09)$ \\
9 & Sleep disturbance & $17(51.51)$ & $3(9.09)$ \\
10 & Sharpness absent & $9(27.27)$ & 0 \\
11 & Cognition absent & $15(45.45)$ & $4(12.12)$ \\
12 & Interpersonal discard & $6(18.2)$ & $2(6.1)$ \\
\hline
\end{tabular}

were reduced after vortioxetine treatment. Other MSE components such as anhedonia, insight, judgment intact, abstract thinking, and pessimism were also reduced from baseline to the end of the treatment (Table 3).

\section{Discussion}

Vortioxetine has been studied for the treatment of depression both as monotherapy and as part of combination therapy to increase the response and remission rate. ${ }^{16,17}$ These studies have established vortioxetine as a valid option for the treatment of depression. However, in Indian scenario, limited outcome research and accessibility to data leads to less thorough knowledge of nature of research problem, and specific studies in Indian patients with depression are required.

The findings of the present prescription review demonstrate that all 33 patients with MDD and anxiety symptoms who received vortioxetine as monotherapy or combination therapy showed improvement in the depression total score (MADRS, HDRS) as well as all other secondary efficacy measures (MCPFQ, BDD-YBOCS, and MSE), with minimum adverse effects. At the end of the treatment,

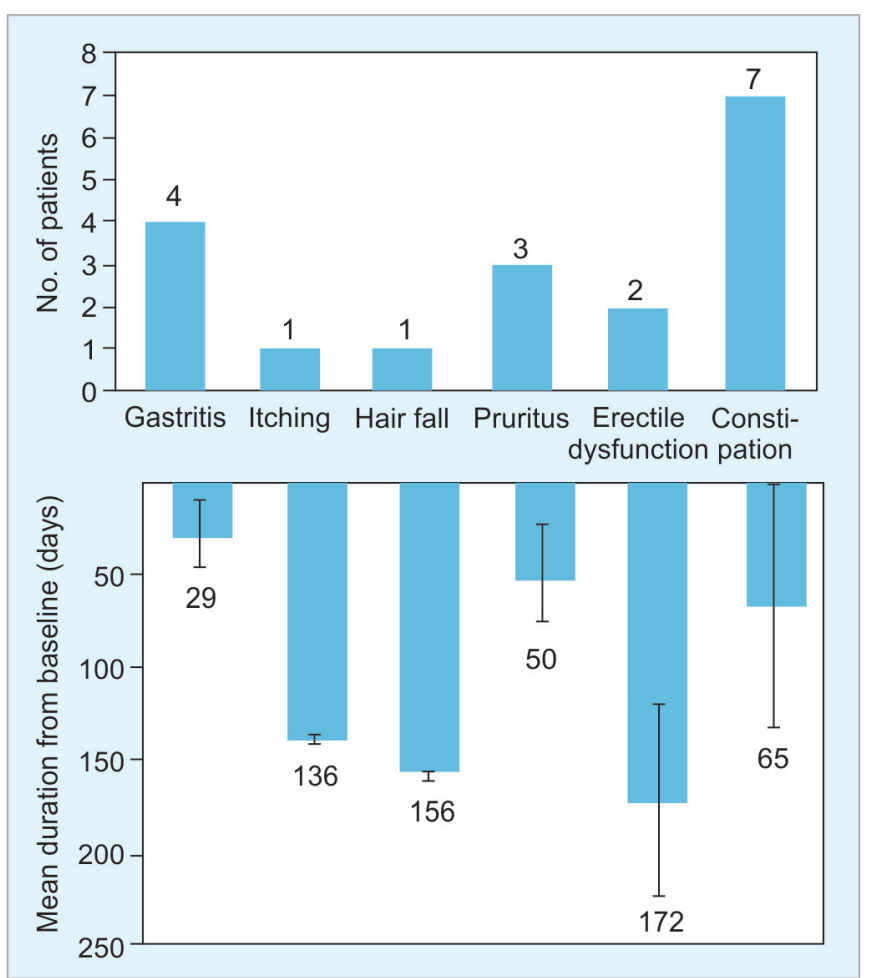

Fig. 3: Adverse effects of vortioxetine therapy and duration of their occurrence in MDD patients

Table 3: Mental status examination (MSE)

\begin{tabular}{llll}
\hline S. No. & Parameters & Baseline MSE (\%) & Final MSE (\%) \\
\hline 1 & Conscious & $33(100)$ & $33(100)$ \\
2 & Nonoriented & $10(33.33)$ & $2(6.06)$ \\
3 & Nonreactive & $16(48.5)$ & $2(6.06)$ \\
4 & Anhedonia present & $20(60.6)$ & 0 \\
5 & Insight absent & $1(3.03)$ & 0 \\
6 & Judgment intact absent & $3(9.09)$ & 0 \\
7 & Abstract thinking absent & $1(3.03)$ & 0 \\
8 & Pessimism present & $3(9.09)$ & 0 \\
\hline
\end{tabular}

cognitive function was improved in $85.7 \%$ of patients with only two still complaining about nonorientation. The overall findings support the potential for the rapid efficacy and acceptable tolerability of vortioxetine in treating depression and anxiety in this population.

Since comorbid anxiety disorders are observed in a substantial proportion of MDD patients (with ranges of $\sim 30-50 \%$ reported depending upon the population sampled), the efficacy of vortioxetine on the physical symptoms of depression in MDD patients with marked coexisting anxiety symptoms is encouraging. ${ }^{18}$ Vortioxetine was reported to be efficacious in reducing both depression and anxiety in MDD patients with high levels of anxiety by a meta-analysis of randomized clinical trials. ${ }^{13}$ In this retrospective prescription review, the severity of depressive and anxiety symptoms for vortioxetine-treated patients, as measured by MADRS, MCPFQ, HDRS, BDD-YBOCS, and MSE scores, improved significantly at the end of the treatment. Together with the results reported here on the physical symptoms of depression, vortioxetine also seems to be a rational treatment option in patients with 
MDD and high anxiety, who often do not respond satisfactorily to alternative antidepressant therapy.

Apart from treating MDD, an effective antidepressant therapy also reduces the risk of relapse or recurrence of the depression. However, long-term efficacy of an antidepressant depends on the patient's compliance. It was estimated that only 10\% MDD patients were adequately adherent to the dosage of prescribed antidepressants and duration of treatment. ${ }^{19}$ In this review, all the 33 patients were prescribed either with 5 or $10 \mathrm{mg}$ of vortioxetine initially as monotherapy (57.6\%) or combination therapy (42.4\%), but by the end of the study, 7 (21.2\%) patients were on $20 \mathrm{mg}$ dose of vortioxetine and more than $72 \%$ patients were receiving vortioxetine monotherapy. The recommended starting daily dose is $10 \mathrm{mg}$ for adults and $5 \mathrm{mg}$ for elderly adults (i.e., those over 65 years of age). ${ }^{20}$ Peak plasma concentrations of 9 to $33 \mathrm{ng} / \mathrm{mL}$ reach in 7 to 11 hours following multiple administrations of 5 to $10 \mathrm{mg} /$ day of vortioxetine.

The most common adverse effects observed in this retrospective prescription review were constipation and gastritis. This was in accordance with previous meta-analysis in which the most common adverse effects reported were gastrointestinal in nature (e.g., nausea, constipation, and vomiting). In $32 \%$ of patients on vortioxetine ( $20 \mathrm{mg}$ daily), nausea appeared frequently and dose dependently. ${ }^{21}$ They also reported the lower rate of spontaneously self-reported sexual dysfunction in patients receiving vortioxetine. SSRIs are one of the most used first-line antidepressant agents as they are considered to have more favorable adverse effect profiles compared to other antidepressants. ${ }^{20}$ Still, they exhibit substantially increased risk for certain highly undesirable adverse effects, such as sexual dysfunction, which may contribute to medication nonadherence. In this retrospective prescription review, only two patients complained of sexual dysfunction at the end of treatment. In 2014, a study has indicated a potential for vortioxetine to reverse sexual dysfunction caused by SSRI therapy while maintaining clinical efficacy. ${ }^{22}$

The beneficial effect of vortioxetine treatment on low mood, lack of interest, lassitude, subjective sleep quality, and daytime somnolence was also documented in this retrospective review. Previous study has reported an improvement in sleep continuity and efficiency after vortioxetine therapy without any influence on diurnal alertness and daytime functioning. ${ }^{23}$ The significant improvement in sleep by vortioxetine was associated with the amelioration of depressive symptoms in MDD patients. ${ }^{24}$

Vortioxetine also improves cognitive functions in MDD patients such as functional capacity, attention, and executive function. ${ }^{25,26}$ In this review, vortioxetine improved cognitive functions in the majority of patients along with the reversal of psychological dysfunctions such as nonreactiveness, anhedonia, and pessimism. Mclntyre et al. reported that the vortioxetinemediated improvement of cognitive function was independent of its antidepressant efficacy. ${ }^{27}$ Vortioxetine has also demonstrated an improvement in a measure of anhedonia, reward, and motivation. ${ }^{24,28}$ Beneficial effects of vortioxetine on physical, psychological, and cognitive functions might provide the MDD patients with best chance for a full functional recovery.

This prescription review has highlighted the treatment benefits associated with vortioxetine in MDD patients. However, the retrospective design and the small size of the cohort preclude clear conclusions on the efficacy and tolerability of vortioxetine in MDD patients with depressive and anxiety symptoms.

\section{Conclusion}

In conclusion, our results have clearly shown the effectiveness, and tolerability of vortioxetine for MDD patients with depressive and anxiety symptoms in Indian scenario. The present prescription review warrants subsequent, adequately powered, randomized controlled trials for generalizability in Indian population.

\section{ACKNOWLEDGMENTS}

The authors want to acknowledge WorkSure India for medical writing assistance

\section{References}

1. Arvind BA, Gururaj G, Loganathan S, et al. Prevalence and socioeconomic impact of depressive disorders in India: multisite population-based cross-sectional study. BMJ Open 2019;9(6):e027250. DOI: 10.1136/bmjopen-2018-027250.

2. Ishak WW, Balayan K, Bresee C, et al. A descriptive analysis of quality of life using patient-reported measures in major depressive disorder in a naturalistic outpatient setting. Qual Life Res 2013;22(3):585-596. DOI: 10.1007/s11136-012-0187-6.

3. Birnbaum HG, Kessler RC, Kelley D, et al. Employer burden of mild, moderate, and severe major depressive disorder: mental health services utilization and costs, and work performance. Depress Anxiety 2010;27(1):78-89. DOI: 10.1002/da.20580.

4. Olesen J, Gustavsson A, Svensson M, et al. The economic cost of brain disorders in Europe. Eur J Neurol 2012;19(1):155-162. DOI: 10.1111/j.1468-1331.2011.03590.x.

5. Gasse C, Petersen L, Chollet J, et al. Pattern and predictors of sick leave among users of antidepressants: a Danish retrospective register-based cohort study. J Affect Disord 2013;151(3):959-966. DOI: 10.1016/j.jad.2013.08.015.

6. Krol M, Papenburg J, Koopmanschap M, et al. Do productivity costs matter? Pharmacoeconomics 2011;29(7):601-619. DOI: 10.2165/11539970-000000000-00000.

7. Thomas $\mathrm{CM}$, Morris $\mathrm{S}$. Cost of depression among adults in England in 2000. Br J Psychiatry 2003;183(6):514-519. DOI: 10.1192/bjp.183.6.514.

8. Ho N, Sommers MS, Lucki I. Effects of diabetes on hippocampal neurogenesis: links to cognition and depression. Neurosci Biobehav Rev 2013;37(8):1346-1362. DOI: 10.1016/j.neubiorev.2013.03.010.

9. Montgomery SA, Gommoll CP, Chen C, et al. Efficacy of levomilnacipran extended-release in major depressive disorder: pooled analysis of 5 double-blind, placebo-controlled trials. CNS Spectr 2015;20(2):148156. DOI: $10.1017 /$ S1092852914000273.

10. Pence BW, O'Donnell JK, Gaynes BN. The depression treatment cascade in primary care: a public health perspective. Curr Psychiatry Rep 2012;14(4):328-335. DOI: 10.1007/s11920-012-0274-y.

11. Ansseau M, Demyttenaere K, Heyrman J, et al. Objective: remission of depression in primary care The Oreon Study. Eur Neuropsychopharmacol 2009;19(3):169-176.DOI: 10.1016/j.euroneuro. 2008.10.003.

12. Sanchez C, Asin KE, Artigas F. Vortioxetine, a novel antidepressant with multimodal activity: review of preclinical and clinical data. Pharmacol Ther 2015;145:43-57. DOI: 10.1016/j.pharmthera.2014.07.001.

13. Baldwin DS, Florea I, Jacobsen PL, et al. A meta-analysis of the efficacy of vortioxetine in patients with major depressive disorder (MDD) and high levels of anxiety symptoms. J Affect Disord 2016;206:140-150. DOI: 10.1016/j.jad.2016.07.015.

14. Berhan A, Barker A. Vortioxetine in the treatment of adult patients with major depressive disorder: a meta-analysis of randomized double-blind controlled trials. BMC Psychiatry 2014;14(1):276. DOI: 10.1186/s12888-014-0276-x.

15. Thase ME, Mahableshwarkar AR, Dragheim M, et al. A metaanalysis of randomized, placebo-controlled trials of vortioxetine for the treatment of major depressive disorder in adults. Eur 
Neuropsychopharmacol 2016;26(6):979-993. DOI: 10.1016/ j.euroneuro.2016.03.007.

16. Gonda X, Sharma SR, Tarazi FI. Vortioxetine: a novel antidepressant for the treatment of major depressive disorder. Expert Opin Drug Discov 2019;14(1):81-89. DOI: 10.1080/17460441.2019.1546691.

17. Koesters M, Ostuzzi G, Guaiana G, et al. Vortioxetine for depression in adults. Cochrane Database Syst Rev 2017;7:CD011520. DOI: 10.1136/ bmjopen-2018-027250.

18. Kessler RC, Sampson NA, Berglund P, et al. Anxious and non-anxious major depressive disorder in the World Health Organization World Mental Health Surveys. Epidemiol Psychiatr Sci 2015;24(3):210-226. DOI: $10.1017 /$ S2045796015000189.

19. Clevenger SS, Malhotra D, Dang J, et al. The role of selective serotonin reuptake inhibitors in preventing relapse of major depressive disorder. Ther Adv Psychopharmacol 2018;8(1):49-58. DOI: $10.1177 / 2045125317737264$.

20. Al-Sukhni M, Maruschak NA, Mclntyre RS. Vortioxetine: a review of efficacy, safety and tolerability with a focus on cognitive symptoms in major depressive disorder. Expert Opin Drug Safety 2015;14(8):12911304. DOI: $10.1517 / 14740338.2015 .1046836$.

21. Citrome L. Vortioxetine for major depressive disorder: a systematic review of the efficacy and safety profile for this newly approved antidepressant - what is the number needed to treat, number needed to harm and likelihood to be helped or harmed? Int J Clin Pract 2014;68(1):60-82. DOI: 10.1111/ijcp.12350.

22. Jacobsen PL, Mahableshwarkar AR, Chen Y, et al. Effect of vortioxetine vs. escitalopram on sexual functioning in adults with well-treated major depressive disorder experiencing SSRI-induced sexual dysfunction. J Sex Med 2015;12(10):2036-2048. DOI: 10.1111/jsm.12980.

23. Liguori C, Ferini-Strambi L, Izzi F, et al. Preliminary evidence that vortioxetine may improve sleep quality in depressed patients with insomnia: a retrospective questionnaire analysis. $\mathrm{Br} \mathrm{J}$ Clin Pharmacol 2019;85(1):240-244. DOI: 10.1111/bcp.13772.

24. Cao B, Park C, Rosenblat JD, et al. Changes in sleep predict changes in depressive symptoms in depressed subjects receiving vortioxetine: An open-label clinical trial. J Psychopharmacol 2019;33(11):1388-1394. DOI: $10.1177 / 0269881119874485$.

25. Christensen MC, Florea I, Lindsten A, etal. Efficacy of vortioxetine on the physical symptoms of major depressive disorder. J Psychopharmacol 2018;32(10):1086-1097. DOI: 10.1177/0269881118788826.

26. Mahableshwarkar AR, Zajecka J, Jacobson W, et al. A randomized, placebo-controlled, active-reference, double-blind, flexible-dose study of the efficacy of vortioxetine on cognitive function in major depressive disorder. Neuropsychopharmacology 2015;40(8):20252037. DOI: $10.1038 / \mathrm{npp} .2015 .52$.

27. McIntyre RS, Lophaven S, Olsen CK. A randomized, double-blind, placebo-controlled study of vortioxetine on cognitive function in depressed adults. Int J Neuropsychopharmacol 2014;17(10):1557-1567. DOI: $10.1017 /$ S1461145714000546.

28. Subramaniapillai M, Mansur RB, Zuckerman H, et al. Association between cognitive function and performance on effort based decision making in patients with major depressive disorder treated with Vortioxetine. Compr Psychiatry 2019;94:152113. DOI: 10.1016/j. comppsych.2019.07.006. 